\title{
Erratum to: Combined posterolateral corner and acute anterior cruciate ligament injuries in an adolescent cohort: a magnetic resonance imaging analysis
}

\author{
Kenneth Aaron Shaw ${ }^{1} \cdot$ Brian S. Dunoski $^{2} \cdot$ Neil J. Mardis $^{2}$ • Donna M. Pacicca ${ }^{3}$
}

Published online: 4 January 2016

(C) SICOT aisbl 2016

Erratum to: International Orthopaedics (SICOT)

DOI 10.1007/s00264-015-3026-x

Reason: The reference number 20 has the wrong name of the first author.

Corrected version: Reference 20. (incorrect: Monadio MB), Correct: Bonadio MB

$\triangle$ Kenneth Aaron Shaw

shaw.aaron82@gmail.com

1 Department of Orthopaedic Surgery, Dwight D. Eisenhower Army Medical Center, 300 East Hospital Road, Fort, Gordon, GA 30905, USA

2 Department of Radiology, Children's Mercy Hospital, Kansas City, MO, USA

3 Division of Orthopaedic Surgery, Children's Mercy Hospital, Kansas City, MO, USA 